\title{
Programa de intervención escolar sobre publicidad y consumo responsable
}

\author{
Judit Miralles Argilés \\ al345689@uji.es \\ Beatriz Dámaso Vega \\ damaso@uji.es
}

\section{Resumen}

Introducción: La publicidad y los medios de comunicación masivos o mass media tienen un papel importante en la adquisición de hábitos de conducta en edades escolares, siendo las más afectadas por ser fases de descubrimiento y captación de nuevas experiencias y hábitos de vida. En este contexto, la enfermera escolar tiene un papel importante al ser una de sus funciones la promoción y educación para la salud, ya que, mediante la incorporación de conocimientos y directrices para el consumo responsable, se puede conseguir el cambio de estas prácticas inadecuadas hacia unas actitudes que permitan una salud óptima en un futuro.

Objetivos: Diseñar un programa de intervención dirigido a escolares y a sus familias que permita concienciar acerca de los malos hábitos que son adquiridos a causa de la publicidad engañosa y los medios de comunicación.

Metodología: Realización de una búsqueda de la literatura en distintas bases de datos y otras páginas web de interés y obtención únicamente de un programa sobre publicidad y consumo responsable impartido en escuelas madrileñas.

Desarrollo del proyecto: El programa tiene una duración de cinco sesiones, cuatro de ellas adaptadas a los cursos académicos a intervenir y la quinta dirigida a los tutores de los alumnos.

Conclusión: Con un único programa hallado en Madrid en las escuelas acerca de la publicidad, ningún programa llevado a cabo en la Comunidad Valenciana relacionado con este tema, ni tampoco ningún programa en el ámbito internacional publicado, se crea uno de elaboración propia. Tras impartir este programa, se pretende conseguir un cambio cuantitativo de conocimientos y un cambio cualitativo en las actitudes que conduzca a un cambio real en las conductas.

Palabras clave: medios de comunicación, promoción de la salud, servicios de salud escolar, servicios de enfermería escolar.

\section{Abstract}

Introduction: Advertising and the Mass Media have an important role in the acquisition of behavioural habits in children. These are the most affected because they live in an age of discovery and capture of new experiences and of habits of lifetime. In this context, the school nurses play an important role as 
one of their functions is health promotion and education, since it is possible, through the incorporation of knowledge and guidelines for responsible consumption, to achieve the change of these inappropriate practices towards attitudes that allow optimal health in the future.

Aims: Design an intervention program aimed at school children and their families to raise awareness about the bad habits that are acquired due to misleading advertising and the media.

Methodology: Conducting a literature search in different databases and other web pages of interest, obtaining only one program about advertising and responsible consumption taught in schools in Madrid.

Development of the project: Duration of the program five sessions, four of them adapted to the academic courses to intervene and the fifth directed to the tutors of the students.

Conclusion: With a single program found in Madrid in schools about advertising, no program carried out in the Valencian Community related to this topic, nor at a published international level, one created by itself was created. After imparting this program, the aim is to achieve a quantitative change of knowledge and a qualitative change in attitudes, leading to a real change in behaviors.

Keywords: Media, Health Promotion, School Health Services, School Nursing Services.

\section{Introducción}

La etapa escolar es aquella en la que el niño adquiere el aprendizaje de ciertas conductas que marcarán los hábitos, las costumbres y la salud que llevará durante su vida (Unicef s. f.). En esta etapa se forma la personalidad, resultado del proceso de socialización mediante el cual se aprende a distinguir lo positivo de lo negativo (Esmaeilzadeh, Ashrafi-rizi,Shahrzadi y Mostafavi 2018) y participan tres agentes: adultos, iguales y medios de comunicación. Debido a su influencia, el niño puede adquirir conductas inapropiadas que deben ser modificadas.

Es en este momento cuando entra en juego el papel de la enfermería en la educación y la promoción de la salud (Federación de Enseñanza de CC. OO. de Andalucía 2010).

La enfermera escolar tiene como objetivo ofrecer cuidados y colaborar para alcanzar el máximo crecimiento y bienestar físico, mental y social en el colectivo educativo (Ramage y Moorley 2019), asumiendo diversas funciones (docente, gestora e investigadora y asistencial), donde lleva a cabo programas de educación para la salud con el propósito de mejorar los estilos de vida y dar continuidad a los conocimientos para convertirlos en hábitos (Vela Ortiz, Ávila Olivares y Candela Fernández 2009).

La enfermera escolar debe, además, estar actualizada en los problemas que atañen a los escolares a causa de las nuevas tecnologías, el progreso y la cultura, como los medios de comunicación y su influencia en la sociedad, que influyen tanto en la forma de pensar como de actuar de las personas (Hospital de día 2014).

A día de hoy, existe una regulación específica de ciertos productos como el tabaco y el alcohol; sin embargo, existen una serie de vacíos legales, ya que se continúan colocando estos productos en revistas, programas y televisión. 
Las herramientas que se encargan de difundir contenido diverso al público general y tienen como objetivo satisfacer las necesidades de los espectadores, ya sea con un fin económico o social, son los mass media (Insituto Internacional Español de Marketing digital s. f.) y es el concepto llamado media advocacy el que pretende utilizar los mass media para prevenir problemas de salud mediante la promoción de hábitos saludables (Wallack 1993; Reuter et al. 2019).

A pesar de eso, la publicidad afecta de manera negativa tanto a niños como a jóvenes, ya que no son capaces de comprender las técnicas persuasivas que utiliza la publicidad y están expuestos a comerciales de comida basura y a la proyección de violencia (González Hidalgo y Atalah Samur 2011; Cornelius, Whitaker-Brown, Neely, Kennedy y Okoro 2019), acarreando una serie de problemas como la obesidad, los trastornos alimentarios y la pérdida de valores (Dominici Jr. Valdez 2017).

La TV Worldwide afirma que los niños españoles entre 4 y 12 años pasan una media de 2 horas y 38 minutos frente a la TV (un 11,2 \% más que hace 7 años) (Parra s.f.). Otro estudio de la Universidad de Michigan revela que los niños que abusan de la TV tienen más problemas en el colegio y padecen más hábitos nocivos (García-Allen s.f.).

En la Comunidad de Madrid, mediante la Dirección General de Comercio y Consumo, se lleva a cabo cada año un programa de educación en consumo que pretende fomentar actitudes y hábitos saludables en el colectivo educativo (Portal del Consumidor de la Comunidad de Madrid 2018). Todavía se desconoce si la intervención en Madrid ha sido efectiva, pues aún no ha sido evaluada y es por esto, y por la influencia nociva de los medios de comunicación, por lo que se diseña un programa de intervención llevado a cabo en la Comunidad Valenciana por la enfermera escolar, porque reforzando la educación se pueden promover hábitos adecuados.

\section{Objetivos}

El desarrollo de este programa consta de los siguientes objetivos:

-Objetivo general: diseñar un programa de intervención dirigido a escolares de 6 a 14 años y sus familias para concienciar sobre los malos hábitos adquiridos a través de la publicidad engañosa y los medios de comunicación.

-Objetivos específicos: fomentar los hábitos saludables que promuevan una reflexión acerca del consumo responsable y evaluar todos los elementos que componen o participan en el programa.

\section{Metodología}

Las palabras clave utilizadas para realizar la búsqueda bibliográfica son: medios de comunicación, educación escolar, promoción de la salud y enfermería escolar. En la tabla 1 se muestran los descriptores empleados. 
Tabla 1

Palabras clave y descriptores empleados

\begin{tabular}{|c|c|c|c|}
\hline Palabras clave & $\begin{array}{l}\text { Palabras } \\
\text { (inglés) }\end{array}$ & DeCS & $\mathrm{MeSH}$ \\
\hline $\begin{array}{l}\text { Medios } \\
\text { comunicación }\end{array}$ & Media & $\begin{array}{ll}\text { Medios } & \text { de } \\
\text { comunicación }\end{array}$ & $\begin{array}{l}\text { Communications } \\
\text { media }\end{array}$ \\
\hline $\begin{array}{l}\text { Educación } \\
\text { escolar }\end{array}$ & School education & $\begin{array}{l}\text { Servicios de salud } \\
\text { escolar }\end{array}$ & $\begin{array}{l}\text { School health } \\
\text { services }\end{array}$ \\
\hline $\begin{array}{l}\text { Promoción de la } \\
\text { salud }\end{array}$ & Health promotion & $\begin{array}{l}\text { Promoción de la } \\
\text { salud }\end{array}$ & Health promotion \\
\hline $\begin{array}{l}\text { Enfermería } \\
\text { escolar }\end{array}$ & School nursing & $\begin{array}{ll}\text { Servicios } & \text { de } \\
\text { enfermería } & \\
\text { escolar } & \end{array}$ & School nursing \\
\hline
\end{tabular}

La búsqueda ha sido realizada entre los meses de febrero y mayo del 2019, en las siguientes bases de datos: Pubmed, Portal Regional de la BVS, SciELO, Biblioteca Cochrane y Epistemonikos, utilizando los DeCS y los MeSH combinando los operadores booleanos $A N D$ y $O R$ y restringiendo la búsqueda a los últimos 10 años, seleccionando el español y el inglés como idiomas de elección.

Al no obtener ningún resultado de utilidad, se realiza una búsqueda adicional en Internet encontrando numerosos artículos en diferentes páginas web que justifican y respaldan el desarrollo del programa: OMS 2013; Generalitat Valenciana 2018; Chacón Medina 2005; Mañas-Viniegra 2018; Comité de Bioética de Aragón 2018 y Ruiz s. f.

La elaboración del programa de salud tiene en cuenta unos criterios tanto de inclusión como de exclusión:

- $\quad$ Criterios de inclusión escolares:

- Consentimiento informado de participación firmado por los padres/madres/tutores.

- Permiso para llevar a cabo el programa por Conselleria de Sanitat, Dirección del centro escolar y Asociación de Padres y Madres de Alumnos (AMPA).

- Criterios de exclusión escolares:

- No autorización de padres o tutor legal para participar en el programa.

- Incapacidad física o mental que dificulte la comprensión e interpretación de los contenidos.

- $\quad$ Criterios de inclusión padres:

- Ser padre/madre/tutor legal del alumno matriculado en educación primaria o secundaria.

- No presentar incapacidad física o mental que imposibilite una adecuada comprensión y asimilación de los contenidos del programa o asistencia a las sesiones.

El programa se llevará a cabo en cinco sesiones, cuatro de ellas adaptadas a los cursos a intervenir $\left(1 .^{\circ}\right.$ y $2 .^{\circ}$ de primaria, $3 .^{\circ}$ y $4 .^{\circ}$ de primaria, $5 .^{\circ}$ y $6 .^{\circ}$ de primaria y $1 .^{\circ}$ y $2 .^{\circ}$ de ESO) y una dirigida a padres/madres/tutores legales de los alumnos, que será la primera en impartirse. 
Para poder ejecutar el programa se solicitará la autorización de la Conselleria, Dirección del centro y AMPA, adjuntando el resumen del programa de intervención.

Además, se tendrá en cuenta al profesorado del centro escolar y se enviará una carta de presentación para ofrecer información acerca del programa.

En educación primaria el programa se impartirá en el horario de la asignatura Valores sociales y cívicos y en educación secundaria se impartirá en el horario de la asignatura Valores éticos.

El programa será llevado a cabo por la enfermera escolar, que puede estar o no ejerciendo sus funciones en el centro, y se requerirá la presencia de los docentes durante el programa para un correcto desarrollo del mismo.

\section{Desarrollo del proyecto}

El programa constará de 5 sesiones:

La sesión 1, "Los mayores también deben ser informados», irá dirigida a padres/madres/tutores de los alumnos; su objetivo principal se centra en concienciar acerca de la adquisición de malos hábitos, ya que es responsabilidad de la familia y comienza en el hogar.

La sesión 2, «La salud y los medios», irá dirigida a los alumnos de $1 .^{\circ}$ y $2 .^{\circ}$ de primaria y consta de dos talleres enfocados principalmente a reflexionar acerca de los hábitos alimentarios y concienciar sobre los hábitos erróneos que se pueden adquirir a causa de la publicidad.

La sesión 3, «Hacia un consumo responsable», irá dirigida a los alumnos de $3 .^{\circ}$ y $4 .^{\circ}$ de primaria; esta sesión consta de dos talleres con actividades enfocadas a valorar la importancia de no derrochar agua y electricidad y enseñar a realizar compras responsables y evitar así el consumismo.

La sesión 4 , «Consumo lo que veo», irá dirigida a los alumnos de $5 .^{\circ}$ y $6 .^{\circ}$ de primaria. Los talleres de los que consta irán enfocados a reflexionar cómo los medios de comunicación influyen en la compra de ciertos productos y a aprender la importancia de la lectura de la letra pequeña de los anuncios (Thomas et al. 2018).

La sesión 5 , «¡Yo contra la publicidad!», irá dirigida a los alumnos de $1 .^{\circ}$ y $2 .^{\circ}$ de ESO. Consta de dos talleres con los objetivos de iniciar al alumnado en la problemática de los trastornos de la conducta alimentaria y conocer las técnicas que utiliza la publicidad para captar la atención del público (Golan y Ahmad 2018).

La duración del programa de intervención será de 4 semanas, con una periodicidad de 2 años; el mes en el que se realizará el programa puede variar, aunque es normal que se realice entre los meses de noviembre, enero o febrero (no coincidentes con evaluaciones finales y con disponibilidad del profesorado). Meses antes de impartir el programa (mayo-junio) se propondrá el programa tanto a la escuela como a Conselleria.

Cada sesión requiere de dos horas aproximadamente para la realización de los talleres por curso, impartidos por dos enfermeras escolares a la misma hora en clases diferentes.

De esta manera, se consigue una continuidad del programa, al realizarse cada 2 años y los alumnos, al cambiar de curso, participan en todas las sesiones. 
Tabla 2

Cronograma de realización de las sesiones del programa

\begin{tabular}{|l|l|l|l|l|}
\hline Sesiones & Semana 1 & Semana 2 & Semana 3 & Semana 4 \\
\hline Sesión 1: Padres/madres/tutores & & & & \\
\hline Sesión 2: $1 .^{\circ}$ y $2 .^{\circ}$ primaria & & & & \\
\hline Sesión 3: $3 .^{\circ}$ y $4 .^{\circ}$ primaria & & & & \\
\hline Sesión 4: $5 .^{\circ}$ y $6 .^{\circ}$ primaria & & & & \\
\hline Sesión 5: $1 .^{\circ}$ y $2 .^{\circ}$ ESO & & & & \\
\hline
\end{tabular}

La evaluación del programa se lleva a cabo siguiendo el esquema clásico de Donabedian (1980, citado en Rodríguez-Pérez 2014):

-Estructura o diseño del proyecto: se considera la correcta adaptación de los talleres a la edad del estudiantado y la adecuación del programa a los objetivos que se quieren alcanzar.

-Proceso del programa: se valora si el aula es adecuada (material fungible adecuado, iluminación, sonido...) y la implicación del alumnado durante las sesiones, así como la duración de los talleres, opinión del profesorado acerca del desarrollo del programa y la captación de la atención del estudiantado.

-Resultados esperados al finalizar el programa (Rojas 2004): la evaluación consiste en una memoria final de todos los elementos que componen o participan en el programa mediante tres rúbricas, una al inicio de los talleres, otra al finalizar el taller y otra durante el primer año del programa tras recibir las sesiones, que permitirán apreciar la participación del alumnado, la opinión del profesorado y si las actividades han sido correctamente adaptadas a las edades de los estudiantes entre otras, con el objetivo de mejorar ciertos aspectos del programa que se quiere impartir en los próximos dos años y darle una continuidad (anexos 1-3).

De esta manera se consigue un cambio cuantitativo de conocimientos al cotejar las nociones de los escolares cada año y un cambio cualitativo en las actitudes con el cambio real en sus conductas mediante la valoración de sus habilidades y de lo aprendido en los talleres.

\section{Conclusión}

Tras analizar el contenido de diversos artículos y páginas web relacionados con el tema a tratar, se desarrolla un programa de intervención dirigido a escolares de entre 6 y 14 años y a sus familias, llevado a cabo por la enfermera escolar, que consta de talleres y actividades adecuados a cada edad, con los que se espera que se modifiquen las conductas inapropiadas y se adquieran ciertas competencias, para llevar una vida saludable en lo que se refiere a la influencia que ejercen los medios de comunicación. 
La educación para la salud tiene que ser capaz de responder con nuevos planteamientos pedagógicos a una realidad tecnológica que avanza rápidamente y que nos reta a trabajar de manera diferente otros aspectos educativos como el consumo responsable y la influencia de la publicidad engañosa.

La educación escolar debe centrarse en las necesidades actuales, dejando de lado la transmisión de conocimientos memorísticos y anticuados y desconectados de los intereses de los escolares. Por ello, el programa está diseñado para que los alumnos trabajen en talleres e interactúen entre ellos.

Mediante el desarrollo consecutivo y dinámico de las sesiones, se despierta el interés en los estudiantes, las familias y los docentes hacia el consumo responsable y se reconocen los malos hábitos que se adquieren a causa de la publicidad. Con su participación en este programa, adquieren hábitos saludables que son los objetivos propuestos de este trabajo.

\section{Referencias bibliográficas}

Chacón Medina, Antonio. 2005. «La infancia ante los nuevos medios». Recuperado de: https://www.ugr.es/ sevimeco/revistaeticanet/Numero4/Articulos/Formateados/ Lainfancia.pdf [Consultado el 18 de abril de 2019].

Comité de Bioética de Aragón. 2018. Informe sobre la publicidad engañosa en cuestiones de salud. Recuperado de:

http://www.aragon.es/estaticos/GobiernoAragon/OrganosConsultivos/ComiteBio eticaAragon/Documentos/Informe_Publicidad.pdf [Consultado el 18 de abril de 2019].

Cornelius, Judith B., Charlene Whitaker-Brown, Tamara Neely, Anna Kennedy y Florence Okoro. 2019. «Mobile phone, social media usage, and perceptions of delivering a social media safer sex intervention for adolescents: results from two countries». Adolescent Health, Medicine and Therapeutics, 10: 29-37. Recuperado de: https://doi.org/10.2147/AHMT.S185041 [Consultado el 22 de mayo de 2019]

Dominici Jr. Valdez, Juan Antonio. 2017. Influencia de la Publicidad en los Niños, Niñas, Adolescentes y Jóvenes (parte I). Recuperado de:

https://www.linkedin.com/pulse/influencia-de-la-publicidad-en-los-niños-niñas-yi [Consultado el 18 de abril de 2019].

Generalitat Valenciana. 2018. Cartera de Servicios de Promoción de salud y prevención en el entorno educativo. Curso escolar 2018-2019. Recuperado de: http://www.ceice.gva.es/documents/162783553/162784556/Cat_Serveis_PdS Escola_18_19_cas.pdf/b4d72783-e293-4778-b62f-fb09cf73dd7b [Consultado el 18 de abril de 2019].

Esmaeilzadeh, Somayeh, Hasan Ashrafi-rizi, Leila Shahrzadi y Firozeh Mostafavi. 2018. "A survey on adolescent health information seeking behavior related to high-risk behaviors in a selected educational district in Isfahan». PLOS ONE, 13(11): e0206647. Recuperado de https://doi.org/10.1371/journal.pone.0206647 [Consultado el 22 de mayo de 2019].

Federación de Enseñanza de CC. OO. de Andalucía. 2010. «Influencia de los medios de comunicación en los niños y niñas». Revista digital para profesionales de la enseñanza, 11. Recuperado de:

https://www.feandalucia.ccoo.es/docu/p5sd7575.pdf [Consultado el 18 de abril de 2019].

García-Allen, Jonathan. s. f. "Los efectos negativos de la televisión en los niños». Recuperado de https://psicologiaymente.com/desarrollo/efectos-negativostelevision-ninos-ciencia [Consultado el 18 de abril de 2019]. 
Golan, Moria y Wiessam Abu Ahmad. 2018. «School-based versus after-school delivery of a universal wellness programme - A randomized controlled multiarm trial». Eating Behaviors, 31: 41-47. Recuperado de https://doi.org/10.1016/j.eatbeh.2018.08.003 [Consultado el 22 de mayo de 2019].

González Hidalgo, Catalina y Eduardo Atalah Samur. 2011. «Regulación de la publicidad televisiva de alimentos para prevenir la obesidad infantil». Archivos Latinoamericanos de Nutrición, 61(3). Recuperado de https://www.alanrevista.org/ediciones/2011/3/art-9/ [Consultado el 18 de abril de 2019].

Hospital de día. 2014. "Los medios de comunicación y su influencia en la sociedad». Hospital de día de Salud Mental del Área de Gestión Sanitaria del sur de Córdoba. Recuperado

de http://www.hospitalinfantamargarita.es/saludmentalhdia/?p=699 [Consultado el 18 de abril de 2019].

Instituto Internacional Español de Marketing digital. s. f. "Qué son mass media Definición y características». IIEMD. Recuperado de https://iiemd.com/massmedia/que-son-mass-media [Consultado el 18 de abril de 2019].

Mañas-Viniegra, Luis. 2018. «La autorregulación de las marcas de juegos de azar online a través de su publicidad en tele visión». Methados. Revista de Ciencias Sociales, 6(1): 19-37. Recuperado de: https://www.methaodos.org/revistamethaodos/index.php/methaodos/issue/viewFile/15/18 [Consultado el 18 de abril de 2019].

OMS. 2013 El papel de la escuela. Recuperado de:

https://www.who.int/dietphysicalactivity/childhood_schools/es/ [Consultado el 18 de abril de 2019].

Parra Gil, Mercedes. s. f. «¿Cuánta televisión deben ver?». Salud y corazón, 107. Recuperado de https://fundaciondelcorazon.com/corazon-facil/blog-impulsovital/2677-icuanta-television-deben-ver.htm [Consultado el 18 de abril de 2019].

Portal del Consumidor de la Comunidad de Madrid. 2018. «Formación en Consumo para centros educativos». Recuperado de:

http://www.madrid.org/cs/Satellite?c=Page\&childpagename=PortalConsumidor \%2FPage\%2FPTCS_contenido\&cid=1343064254484\&pagename=PTCS_wrap per [Consultado el 18 de abril de 2019].

Ramage, Colin y Calvin Moorley. 2019. «A narrative synthesis on healthcare students use and understanding of social media: Implications for practice». Nurse Education Today, 77: 40-52. Recuperado de: https://doi.org/10.1016/j.nedt.2019.03.010 [Consultado el 22 de mayo de 2019].

Reuter, Katja, Alicia MacLennan, Namquyen Le, Jennifer B. Unger, Elsi M. Kaiser y Praveen Angyan. 2019. "A Software Tool Aimed at Automating the Generation, Distribution, and Assessment of Social Media Messages for Health Promotion and Education Research». JMIR Public Health and Surveillance, 5(2): e11263. Recuperado de https://doi.org/10.2196/11263 [Consultado el 22 de mayo de 2019].

Rodríguez Pérez, Paz y Marta Grande Arnesto. 2014. Calidad asistencial: Concepto, dimensiones y desarrollo operativo [Internet]. Madrid: Escuela Nacional de Sanidad. Recuperado de:

http://e-spacio.uned.es/fez/eserv/bibliuned:500957/n14-1_Calidad_asistencial.pdf [Consultado el 18 de abril de 2019]

Rojas Montero, Eliana. 2004. «Marco conceptual para la evaluación de programas de salud». Población y Salud en Mesoamérica, 1(2): 1-17. Recuperado de: http://doi.org/10.15517/PSM.V1I2.13935 [Consultado el 6 de mayo de 2019].

Ruiz, Cristina. La influencia de los medios audiovisuales. Recuperado de: https://losrealejos.es/documentos/bienestar-social/dialogodialogo/2015/medios_audiovisuales.pdf [Consultado el 18 de abril de 2019].

Thomas, Samantha L., Amy Bestman, Hannah Pitt, Rebecca Cassidy, Simone McCarthy, Christian Nyemcsok, Sean Cowlishaw y Mike Daube. 2018. «Young people's awareness of the timing and placement of gambling advertising on 
traditional and social media platforms: a study of 11-16-year-olds in Australia».
Harm
Reduction
Journal,
15(1):
51.
Recuperado
de:

https://doi.org/10.1186/s12954-018-0254-6 [Consultado el 22 de mayo de 2019].

Unicef. s.f. El desarrollo infantil y el aprendizaje temprano. Recuperado de https://www.unicef.org/spanish/ffl/03/2.htm [Consultado el 13 de marzo de 2019].

Vela Ortiz, María del Mar, José Antonio Ávila Olivares y Fernando Candela Fernández. 2009. Perfil profesional de la enfermera escolar. Recuperado de: http://www.sce3.com/pubimg/perfilenfescolargandia2009pdf.pdf [Consultado el 18 de abril de 2019].

Wallack, Lawrence. 1993. "Media advocacy and public health: power for prevention». Sage Publications. Recuperado de https://us.sagepub.com/en-us/nam/mediaadvocacy-and-public-health/b ook4219\#description [Consultado el 18 de abril de 2019]. 


\section{Anexo 1: Rúbrica de evaluación inicial de las sesiones}

\begin{tabular}{|c|c|c|c|c|c|}
\hline & Excelente (2) & Muy bueno $(1,5)$ & Aceptable $(0,75)$ & Insuficiente $(0,25)$ & TOTAL \\
\hline $\begin{array}{l}\text { Conocimientos } \\
\text { previos del } \\
\text { alumnado sobre el } \\
\text { tema }\end{array}$ & $\begin{array}{l}\text { Los conocimientos } \\
\text { previos del alumnado } \\
\text { sobre el tema son muy } \\
\text { amplios. }\end{array}$ & $\begin{array}{l}\text { Los conocimientos } \\
\text { previos del } \\
\text { alumnado sobre el } \\
\text { tema son más que } \\
\text { aceptables! }\end{array}$ & $\begin{array}{l}\text { Los conocimientos } \\
\text { previos del alumnado } \\
\text { sobre el tema son } \\
\text { básicos. }\end{array}$ & $\begin{array}{l}\text { Los conocimientos } \\
\text { previos del alumnado } \\
\text { sobre el tema son } \\
\text { escasos. }\end{array}$ & \\
\hline $\begin{array}{l}\text { Interés del alumnado } \\
\text { sobre el tema }\end{array}$ & $\begin{array}{l}\text { El alumnado tiene un } \\
\text { gran interés por el } \\
\text { tema. }\end{array}$ & $\begin{array}{l}\text { El alumnado tiene } \\
\text { interés por el tema. }\end{array}$ & $\begin{array}{l}\text { El alumnado tiene } \\
\text { algo de interés por el } \\
\text { tema. }\end{array}$ & $\begin{array}{l}\text { El alumnado tiene } \\
\text { poco interés por el } \\
\text { tema. }\end{array}$ & \\
\hline $\begin{array}{l}\text { Aceptación del } \\
\text { programa por parte } \\
\text { del profesorado }\end{array}$ & $\begin{array}{l}\text { Todo el profesorado } \\
\text { acepta el desarrollo } \\
\text { del programa. }\end{array}$ & $\begin{array}{l}\text { El } 75 \% \text { del } \\
\text { profesorado acepta } \\
\text { el desarrollo del } \\
\text { programa. }\end{array}$ & $\begin{array}{l}\text { El } 50 \% \text { del } \\
\text { profesorado acepta el } \\
\text { desarrollo del } \\
\text { programa. }\end{array}$ & $\begin{array}{l}\text { Menos del } 50 \% \text { del } \\
\text { profesorado acepta el } \\
\text { desarrollo del } \\
\text { programa. }\end{array}$ & \\
\hline $\begin{array}{l}\text { Ambiente para la } \\
\text { realización de las } \\
\text { sesiones }\end{array}$ & $\begin{array}{l}\text { El ambiente para la } \\
\text { realización de las } \\
\text { sesiones es excelente. }\end{array}$ & $\begin{array}{l}\text { El ambiente para la } \\
\text { realización de las } \\
\text { sesiones es muy } \\
\text { adecuado. }\end{array}$ & $\begin{array}{l}\text { El ambiente para la } \\
\text { realización de las } \\
\text { sesiones es adecuado. }\end{array}$ & $\begin{array}{l}\text { El ambiente para la } \\
\text { realización de las } \\
\text { sesiones requiere } \\
\text { modificaciones. }\end{array}$ & \\
\hline
\end{tabular}

\section{Anexo 2: Rúbrica de evaluación final de las sesiones}

\begin{tabular}{|c|c|c|c|c|c|}
\hline & Excelente (2) & Muy bien $(1,5)$ & Bien (1) & Hay que m ejorarlo (0) & TOTAL \\
\hline $\begin{array}{l}\text { Adaptación de los } \\
\text { talleres a la ed ad del } \\
\text { estudiantado }\end{array}$ & $\begin{array}{l}\text { Todos los talleres se adaptan } \\
\text { correctamente a la edad del } \\
\text { estudiantado. }\end{array}$ & $\begin{array}{l}\text { El } 80 \% \text { de los talleres se } \\
\text { adaptan correctamente a la edad } \\
\text { del estudiantado. }\end{array}$ & $\begin{array}{l}\text { E1 } 50 \% \text { de los talleres se } \\
\text { adaptan correctamente a la } \\
\text { edad del estudiantado. }\end{array}$ & $\begin{array}{l}\text { Menos del } 50 \% \text { de los talleres se } \\
\text { adaptan correctamente a la edad } \\
\text { del estudiantado. }\end{array}$ & \\
\hline $\begin{array}{l}\text { Adecuación del } \\
\text { programa a los objetiros } \\
\text { a alcanzar }\end{array}$ & $\begin{array}{l}\text { El programa se adecua al } 100 \% \\
\text { para alcanzar los objetivos. }\end{array}$ & $\begin{array}{l}\text { El programa se adecua al } 75 \% \\
\text { para alcanzar los objetivos. }\end{array}$ & $\begin{array}{l}\text { El programa se adecua al } 50 \% \\
\text { para alcanzar los objetivos. }\end{array}$ & $\begin{array}{l}\text { El programa se adecua menos del } \\
50 \% \text { para alcanzar los objetivos. }\end{array}$ & \\
\hline $\begin{array}{l}\text { Implicación del } \\
\text { alumnado durante las } \\
\text { sesiones }\end{array}$ & $\begin{array}{l}\text { El } 100 \% \text { de los alumnos } \\
\text { participan en todo momento en } \\
\text { los talleres sin tener que pedir su } \\
\text { participación. }\end{array}$ & $\begin{array}{l}\text { El } 75 \% \text { de los alumnos } \\
\text { participan durante el desarrollo } \\
\text { de la sesión sin tener que pedir } \\
\text { su participación. }\end{array}$ & $\begin{array}{l}\text { Más del } 50 \% \text { de la clase } \\
\text { participa en las actividades de } \\
\text { la sesión teniendo que pedir su } \\
\text { colaboración en más de dos } \\
\text { ocasiones. }\end{array}$ & $\begin{array}{l}\text { E1 } 50 \% \text { más de los alumnos no } \\
\text { participan en las actividades de la } \\
\text { clase y se tiene que pedir su } \\
\text { participación en más de tres } \\
\text { ocasiones. }\end{array}$ & \\
\hline $\begin{array}{l}\text { Duración de los talleres } \\
\text { (ajuste al tiempo } \\
\text { establecido) }\end{array}$ & $\begin{array}{l}\text { Todas las partes de la sesión se } \\
\text { ajustan a los tiempos. }\end{array}$ & $\begin{array}{l}\text { Debido a algunas internupciones } \\
\text { se desajusta el tiempo de la } \\
\text { sesión, pero es te se reajusta con } \\
\text { facilidad. }\end{array}$ & $\begin{array}{l}\text { Alguna de las partes de la } \\
\text { sesión no se ajusta } \\
\text { correctamente al tiempo } \\
\text { establecido. }\end{array}$ & $\begin{array}{l}\text { Ninguno de los tiempos } \\
\text { es tablecidos se cumple dur ante las } \\
\text { actividades de la sesión. }\end{array}$ & \\
\hline $\begin{array}{l}\text { Opinión del profesorado } \\
\text { acerca del desarrollo del } \\
\text { programa }\end{array}$ & $\begin{array}{l}\text { Todos los docentes opinan que el } \\
\text { programa se ha des arrollado } \\
\text { adecuadamente. }\end{array}$ & $\begin{array}{l}\text { El } 80 \% \text { de los docentes opinan } \\
\text { que el programa se ha } \\
\text { desarrollado adecuadamente. }\end{array}$ & $\begin{array}{l}\text { E1 } 50 \% \text { de los docentes opinan } \\
\text { que el programa se ha } \\
\text { desarrollado adecuadamente. }\end{array}$ & $\begin{array}{l}\text { Todos los docentes opinan que el } \\
\text { programa no se ha desarrollado } \\
\text { adecuadamente. }\end{array}$ & \\
\hline
\end{tabular}




\section{Anexo 3: Rúbrica de evaluación de las sesiones durante el primer año} del programa tras recibir las sesiones

\begin{tabular}{|c|c|c|c|c|c|}
\hline & Excelente (2) & Muy bueno $(1,5)$ & Aceptable $(0,75)$ & Requiere mejoras $(0,25)$ & TOTAL \\
\hline $\begin{array}{l}\text { Ampliación de } \\
\text { conocimientos del } \\
\text { alumnado sobre el } \\
\text { tema }\end{array}$ & $\begin{array}{l}\text { E1 alumnado ha } \\
\text { conseguido ampliar } \\
\text { sus conocimientos } \\
\text { acerca del tema }\end{array}$ & $\begin{array}{l}\text { El alumnado ha } \\
\text { ampliado bastante sus } \\
\text { conocimientos acerca } \\
\text { del tema. }\end{array}$ & $\begin{array}{l}\text { E1 alumnado ha } \\
\text { conseguido ampliar algo } \\
\text { sus conocimientos acerca } \\
\text { del tema. }\end{array}$ & $\begin{array}{l}\text { El alumnado no ha } \\
\text { ampliado lo suficiente los } \\
\text { conocimientos acerca del } \\
\text { tema. }\end{array}$ & \\
\hline $\begin{array}{l}\text { Alcance de los } \\
\text { objetivos tras un año } \\
\text { del desarrollo }\end{array}$ & $\begin{array}{l}\text { Todos los objetivos } \\
\text { se han alcanzado } \\
\text { tras un año del } \\
\text { desarrollo. }\end{array}$ & $\begin{array}{l}\text { Casi todos los } \\
\text { objetivos se han } \\
\text { al canzado tras un año } \\
\text { del desarrollo. }\end{array}$ & $\begin{array}{l}\text { Se ha alcanzado un } \\
\text { objetivo tras un año del } \\
\text { desarrollo. }\end{array}$ & $\begin{array}{l}\text { No se han conseguido } \\
\text { al canzar los objetivos tras } \\
\text { un año del de sarrollo. }\end{array}$ & \\
\hline $\begin{array}{l}\text { Modificación de } \\
\text { conductas por parte } \\
\text { del alumnadoy } \\
\text { familia }\end{array}$ & $\begin{array}{l}\text { El alumnado y su } \\
\text { familia han } \\
\text { conseguido } \\
\text { modificar todas las } \\
\text { conductas erróneas. }\end{array}$ & $\begin{array}{l}\text { E1 alumnado y su } \\
\text { familia han } \\
\text { conseguido modificar } \\
\text { casi todas las } \\
\text { conductas erróneas. }\end{array}$ & $\begin{array}{l}\text { E1 alumnado y su familia } \\
\text { han conseguido } \\
\text { modificar al guna } \\
\text { conducta errónea. }\end{array}$ & $\begin{array}{l}\text { El alumnado y su familia no } \\
\text { han conseguido modificar } \\
\text { ninguna de las conductas } \\
\text { erróneas. }\end{array}$ & \\
\hline $\begin{array}{l}\text { Opinión del } \\
\text { profesorado sobre la } \\
\text { continuidad del } \\
\text { programa }\end{array}$ & $\begin{array}{l}\text { E1 } 100 \% \text { del } \\
\text { profesorado opina } \\
\text { que el programa } \\
\text { deberia de } \\
\text { continuarse en el } \\
\text { centro. }\end{array}$ & $\begin{array}{l}\text { E1 } 75 \% \text { del } \\
\text { profesorado opina que } \\
\text { el programa deberia } \\
\text { de continuarse en el } \\
\text { centro. }\end{array}$ & $\begin{array}{l}\text { E1 } 50 \% \text { del profesorado } \\
\text { opina que el programa } \\
\text { deberia de continuarse en } \\
\text { el centro. }\end{array}$ & $\begin{array}{l}\text { Menos del } 50 \% \text { del } \\
\text { profesorado opina que el } \\
\text { programa deberia de } \\
\text { continuarse en el centro. }\end{array}$ & \\
\hline
\end{tabular}

